\title{
Untersuchungen über Iodomercurate: Kristallstrukturen von Bis-tetramethylammonium-tetraiodomercurat(II), Tetramethylammoniumpentaiododimercurat(II) und Bis[di(12-krone-4)lithium]-decaiodotetramercurat(II)
}

Investigations on Iodomercurates: Crystal Structures of Bis-tetramethylammonium tetraiodomercurate(II), Tetramethylammonium pentaiododimercurate(II) and Bis[di(12-crown-4)lithium] decaiodotetramercurate(II)

Joachim Pickardt, Sven Wiese und Marina Borowski

Institut für Chemie, Technische Universität Berlin, Straße des 17. Juni 135, D-10623 Berlin

Sonderdruckanforderungen an Prof. Dr. J. Pickardt. E-mail: pickardt@wap0201.chem.tu-berlin.de

Z. Naturforsch. 61b, 935 - 941 (2006); eingegangen am 20. März 2006

Crystals of $\left[\mathrm{Me}_{4} \mathrm{~N}_{2}\left[\mathrm{HgI}_{4}\right]\right.$ (1) and $\left[\mathrm{Me}_{4} \mathrm{~N}\right]\left[\mathrm{Hg}_{2} \mathrm{I}_{5}\right]$ (2), were obtained from solutions of stoichiometric amounts of $\mathrm{HgI}_{2}$ and $\mathrm{Me}_{4} \mathrm{NI}$ in $\mathrm{EtOH}$ or acetone, resp., while crystals of [ $\mathrm{Li}$ (12-crown4) $\left.{ }_{2}\right]_{2}\left[\mathrm{Hg}_{4} \mathrm{I}_{10}\right]$ (3) formed upon interdiffusion of methanolic solutions of $\mathrm{HgI}_{2}$, LiI and of the auxiliary ligand. The crystal structures of the compounds were determined. The $\left[\mathrm{Hg}_{2} \mathrm{I}_{5}\right]^{-}$ion in (2) has a polymeric sheet structure, built up from $\left[\mathrm{Hg}_{2} \mathrm{I}_{6}\right]$ units bridged by $\left[\mathrm{HgI}_{4}\right]$ groups sharing common apices. The $\left[\mathrm{Hg}_{4} \mathrm{I}_{10}\right]^{2-}$ ion in $\mathbf{3}$ consists of four $\left[\mathrm{HgI}_{4}\right]$ tetrahedra sharing common edges. A short survey on the iodomercurate(II) anions known up to now is given and a classification is proposed.

Key words: Mercury, Iodomercurate(II) Anions, Pentaiododimercurate, Decaiodotetramercurate, Crystal Structures

\section{Einleitung}

Im Verlauf von Untersuchungen der Reaktionen von Halogeniden und Pseudohalogeniden der Metalle der 1. und 2. Nebengruppen konnten wir bei der Umsetzung von $\mathrm{HgI}_{2}$ mit Kryptand 222 Kristalle einer Verbindung $[\mathrm{Hg}$ (cryptand 222) $]\left[\mathrm{Hg}_{3} \mathrm{I}_{8}\right]$ mit dem ersten finiten Octaiodotrimercurat $\left[\mathrm{Hg}_{3} \mathrm{I}_{8}\right]^{2-}$ erhalten [1]. Dies führte dazu, dass wir uns näher mit Iodomercuraten beschäftigt haben. Wir fanden, dass sich aus Alkalihalogenomercurat(II)-Lösungen in Gegenwart von Kronenethern häufig Verbindungen mit mehrkernigen Halogenomercurat(II)-Anionen bilden; vermutlich treten dabei „nackte“ HalogenomercuratIonen auf, die zu Polymercurat-Anionen kondensieren.

Bekanntlich löst sich das in Wasser schwer lösliche $\mathrm{HgI}_{2}$ leicht in wässriger KI-Lösung, wofür im allg. formuliert wird, dass dabei komplexe TetraiodomercuratIonen $\left[\mathrm{HgI}_{4}\right]^{2-}$ gebildet werden. Bereits vor über 50 Jahren wurde von Sillén [2] aus spektroskopischen Untersuchungen und Leitfähigkeitsmessungen gefolgert, dass in wässriger Lösung in Abhängigkeit von der Iodidionen-Konzentration folgende Gleichgewich- te vorliegen: $\mathrm{Hg}^{2+} \leftrightarrow[\mathrm{HgI}]^{+} \leftrightarrow \mathrm{HgI}_{2} \leftrightarrow\left[\mathrm{HgI}_{3}\right]^{-} \leftrightarrow$ $\left[\mathrm{HgI}_{4}\right]^{2-}$. In wässrigen Lösungen sollten demnach nur Tri- und Tetraiodomercurat-Anionen existieren, jedoch keine Polymercurate. Dies wurde von Sandström [3] durch Röntgen-Kleinwinkel-Streuungsmessungen und schwingungsspektroskopische Untersuchungen bestätigt. In nichtwässrigen Lösungsmitteln sind die Verhältnisse anders: nach Deacon [4] sollten in $\mathrm{MeOH}$ oder EtOH überwiegend $\left[\mathrm{HgI}_{3}\right]^{-}$-Ionen vorliegen. Hoaper et al. [5] stellten aus $\mathrm{R}_{4} \mathrm{NI}(\mathrm{R}=\mathrm{Me}, \mathrm{Et})$ und $\mathrm{HgI}_{2}$ dagegen in EtOH oder Aceton Mercurate der Zusammensetzung $\left[\mathrm{R}_{4} \mathrm{~N}\right]\left[\mathrm{Hg}_{2} \mathrm{I}_{5}\right]$ und $\left[\left(\mathrm{R}_{4} \mathrm{~N}\right)_{2}\right]\left[\mathrm{Hg}_{3} \mathrm{I}_{8}\right]$ her. Die Verbindungen wurden lediglich elementanalytisch und schwingungsspektroskopisch charakterisiert. Für das postulierte $\left[\mathrm{Hg}_{2} \mathrm{I}_{5}\right]^{-}$-Ion wurden zwei Strukturmodelle vorgeschlagen: entweder zwei über ein gemeinsames Iodid-Ion eckenverknüpfte trigonal-bipyramidalen $\mathrm{HgI}_{5}$-Einheiten oder ein kettenförmiges $\left[\mathrm{Hg}_{4} \mathrm{I}_{10}\right]^{2-}$-Ion aus kantenverknüpften $\left[\mathrm{HgI}_{4}\right]$-Tetraedern. Beide Vorschläge sind falsch, das $\left[\mathrm{Hg}_{4} \mathrm{I}_{10}\right]^{2-}$-Ion wurde von uns in der in dieser Arbeit vorgestellten Verbindung [Li(12-krone-4) $\left.{ }_{2}\right]_{2}\left[\mathrm{Hg}_{4} \mathrm{I}_{10}\right]$ (3) gefunden [6]. Etwa gleichzeitig wurde von Nocke- 
mann et al. die Verbindung Bis(tetrabutylammonium)decaiodotetramercurat(II) dargestellt [7]. Das $\left[\mathrm{Hg}_{2} \mathrm{I}_{5}\right]^{-}$-Ion ist polymer: Wir konnten ein kettenförmig aufgebautes Ion in der von uns dargestellten Verbindung catena-Poly $\{$ di[benzo-15-krone-5)kalium]pentaiododimercurat(II) $\}$ strukturell charakterisieren [8]. In der Verbindung [ $\left.\mathrm{Me}_{4} \mathrm{~N}\right]\left[\mathrm{Hg}_{2} \mathrm{I}_{5}\right]$ (2), die in dieser Arbeit beschrieben wird, sowie dem isostrukturellen $\left[\mathrm{Et}_{4} \mathrm{~N}\right]\left[\mathrm{Hg}_{2} \mathrm{I}_{5}\right]$, liegt ein schichtförmig aufgebautes $\left[\mathrm{Hg}_{2} \mathrm{I}_{5}\right]^{-}$-Ion vor. 2 kristallisiert aus einer Lösung von Tetramethylammoniumiodid und $\mathrm{HgI}_{2} \mathrm{im}$ Molverhältnis $1: 1$ in Aceton. Aus Ethanol konnten wir dagegen bei einem Molverhältnis von 2: 1 das einfache Tetraiodomercurat $\left[\mathrm{Me}_{4} \mathrm{~N}\right]_{2}\left[\mathrm{HgI}_{4}\right]$ (1) erhalten.

Welche Iodomercurat-Anionen sind nun tatsächlich durch Kristallstrukturanalysen charakterisiert? Das Triiodomercurat(II)-Ion $\left[\mathrm{HgI}_{3}\right]^{-}$liegt z. B. in $\left[\mathrm{Me}_{3} \mathrm{~S}\right]\left[\mathrm{HgI}_{3}\right]$ vor [9]; die nahezu planaren $\mathrm{HgI}_{3}-$ Einheiten sind über Hg-I-Kontakte von 352-369 pm zu den Nachbareinheiten zu Ketten verbunden, jedes $\mathrm{Hg}$-Atom ist trigonal-bipyramidal koordiniert. Die Verbindung $\mathrm{K}\left[\mathrm{HgI}_{3}\right] \cdot \mathrm{H}_{2} \mathrm{O}$ enthält dagegen keine $\left[\mathrm{HgI}_{3}\right]$-Einheiten, sondern Ketten eckenverknüpfter $\left[\mathrm{HgI}_{4}\right]$-Tetraeder [10]. Das Tetraiodomercurat(II)Ion, $\left[\mathrm{HgI}_{4}\right]^{2-}$, tritt recht häufig auf, z. B. in $\mathrm{Cs}_{2}\left[\mathrm{HgI}_{4}\right]$ [11]. Interessant ist auch der Komplex $\left[\mathrm{Cs}\left(18-\mathrm{krone}^{-6}\right)\right]\left[\mathrm{HgI}_{4}\right]$, in dem neben $\left[\mathrm{HgI}_{4}\right]^{2-}$-Ionen [Cs(18-krone-6)Cs $]^{2+}$-,Antisandwich-Einheiten“ mit sehr kurzen Cs...Cs-Abständen enthalten sind [12], sowie die Verbindung $\left[\mathrm{La}_{6}(\mathrm{OH})_{8}(\mathrm{O})\left(\mathrm{H}_{2} \mathrm{O}\right)_{24}\right]\left[\mathrm{HgI}_{4}\right]$ mit einem ungewöhnlich großen sechskernigen Komplexkation [8]. Die bekanntesten Tetraiodomercurate, $\mathrm{Cu}_{2} \mathrm{HgI}_{4}$ und $\mathrm{Ag}_{2} \mathrm{HgI}_{4}$, enthalten keine diskreten $\left[\mathrm{HgI}_{4}\right]^{2-}$-Ionen, sondern haben Festkörperstrukturen, die sich z. B. von der Sphalerit-Struktur ableiten lassen [13]. An Polyiodomercurat-Anionen sind bekannt: das Pentaiododimercurat(II)-Ion der formalen Zusammensetzung $\left[\mathrm{Hg}_{2} \mathrm{I}_{5}\right]^{-}$, z. B. in $\left[\mathrm{Me}_{4} \mathrm{~N}\right]\left[\mathrm{Hg}_{2} \mathrm{I}_{5}\right](2$, diese Arbeit) sowie in catena-Poly $\{[\operatorname{di}($ benzo-15-krone5)kalium]pentaiododimercurat(II) \} [8]. Beide enthalten polymere Anionen (s.u.). In der Verbindung $\mathrm{Cs}_{3} \mathrm{HgI}_{5}$ liegen, wie schon aus den formalen Ionenladungen ersichtlich, keine $\mathrm{Hg}_{2} \mathrm{I}_{5}$-Spezies vor, sondern $\left[\mathrm{HgI}_{4}\right]^{2-}$ - und $\mathrm{I}^{-}$-Ionen [11]. [ $\left.\mathrm{Hg}_{2} \mathrm{I}_{6}\right]^{2-}$-Anionen finden sich z. B. im Komplex [Cs(15-krone-5) $]_{2}\left[\mathrm{Hg}_{2} \mathrm{I}_{6}\right]$ [6], ferner in $\left[\mathrm{HgI}(\right.$ diaza-15-krone-5) $]\left[\mathrm{Hg}_{2} \mathrm{I}_{6}\right]$ [14]; letzteres ist interessant, weil kein Alkali- oder Erdalkalimetallion darin enthalten ist. Nach Wells [15] sollen $\left[\mathrm{Hg}_{2} \mathrm{I}_{6}\right]^{2-}$-Anionen sich bevorzugt mit größeren Kationen bilden. Dies konnten wir jedoch nicht be-
Tab. 2. Ausgewählte Bindungslängen (pm) und -winkel $\left({ }^{\circ}\right)$ in $\mathbf{1 .}$

\begin{tabular}{llll}
\hline Hg1-I1 & $278,06(8)$ & Hg1-I2 & $276,34(11)$ \\
Hg1-I3 & $277,16(15)$ & Hg1-I4 & $277,58(15)$ \\
I2-Hg1-I1 & $113,63(3)$ & I3-Hg1-I1 & $107,56(5)$ \\
I4-Hg1-I1 & $108,66(5)$ & I2-Hg1-I3 & $108,07(5)$ \\
I2-Hg1-I4 & $108,13(6)$ & I3-Hg1-I4 & $110,81(4)$ \\
\hline
\end{tabular}

stätigen. Gegenbeispiele sind die oben erwähnten Tetraiodomercurate mit sehr großen Kationen wie dem sechskernigen La(III)-Oxohydrat-Cluster und dem CsSandwich-Kation. Diskrete $\left[\mathrm{Hg}_{3} \mathrm{I}_{8}\right]^{2-}$-Ionen liegen vor in $[\mathrm{Hg}(\mathrm{kryptand} 222)]\left[\mathrm{Hg}_{3} \mathrm{I}_{8}\right]$ [1] und [ $\mathrm{Li}(12-$ krone-4) $\left.{ }_{2}\right]_{2}\left[\mathrm{Hg}_{3} \mathrm{I}_{8}\right]$ [8]. Es handelt sich bei den beiden $\left[\mathrm{Hg}_{3} \mathrm{I}_{8}\right]^{2-}$-Spezies um Isomere. Bei dem ersten ist bemerkenswert, dass in diesem Ion ein dreifach verbrückendes Iodatom vorhanden ist. Das Ion besteht aus drei kantenverknüpften, stark verzerrten $\left[\mathrm{HgI}_{4}\right]$ Tetraedern und kann formal auch als ein $\mathrm{Hg}_{2} \mathrm{I}_{6}$-Ion, das durch $\mathrm{HgI}_{2}$ verbrückt wird, aufgefasst werden. Das zweite Anion hat nur zweifach verbrückende Iodatome. Es besteht ebenfalls aus drei $\left[\mathrm{HgI}_{4}\right]$-Tetraedern, die jedoch alle eine Verbrückung über gegenüberliegende Kanten der Tetraeder aufweisen. Die Verbindung $\mathrm{Cs}_{2} \mathrm{Hg}_{3} \mathrm{I}_{8}$ enthält keine diskreten $\left[\mathrm{Hg}_{3} \mathrm{I}_{8}\right]^{2-}$ Ionen, sondern polymere Schichten eckenverknüpfter $\left[\mathrm{HgI}_{4}\right]$-Tetraeder [11]. Das bisher größte diskrete Iodomercurat-Anion ist das in dieser Arbeit beschriebene Decaiodotetramercurat(II)-Ion, $\left[\mathrm{Hg}_{4} \mathrm{I}_{10}\right]^{2-}$, das wir als $\left[\mathrm{Li}(12-k r o n e-4)_{2}\right]_{2}\left[\mathrm{Hg}_{4} \mathrm{I}_{10}\right]$ (1) erhalten konnten.

Im Folgenden werden die Verbindungen $\left[\mathrm{Me}_{4} \mathrm{~N}\right]_{2}$ $\left[\mathrm{HgI}_{4}\right](\mathbf{1}),\left[\mathrm{Me}_{4} \mathrm{~N}\right]\left[\mathrm{Hg}_{2} \mathrm{I}_{5}\right]$ (2) und [Li(12-krone-4) $\left.{ }_{2}\right]_{2}$ $\left[\mathrm{Hg}_{4} \mathrm{I}_{10}\right]$ (3) beschrieben.

\section{Experimenteller Teil}

Gelbliche Kristalle von Bis-tetramethylammonium-tetraiodomercurat(II), $\left[\mathrm{Me}_{4} \mathrm{~N}_{2}\left[\mathrm{HgI}_{4}\right]\right.$ (1), entstanden aus einer Lösung von $0,201 \mathrm{~g}$ (1 mmol) Tetramethylammoniumiodid und $0,454 \mathrm{~g}$ ( $1 \mathrm{mmol}) \mathrm{HgI}_{2}$ in $20 \mathrm{ml}$ Ethanol.

Hellgelbe Kristalle von Tetramethylammonium-pentaiododimercurat(II), $\left[\mathrm{Me}_{4} \mathrm{~N}\right]\left[\mathrm{Hg}_{2} \mathrm{I}_{5}\right]$ (2), bildeten sich beim langsamen Eindunsten einer Lösung von 0,402 g ( $2 \mathrm{mmol})$ Tetramethylammoniumiodid und $0,454 \mathrm{~g}$ (1 mmol) $\mathrm{HgI}_{2}$ in $40 \mathrm{ml}$ Aceton, das $5 \%$ Wasser enthielt.

Kristalle von Bis[di(12-krone-4)lithium]decaiodotetramercurat(II), [Li(12-krone-4) $\left.)_{2}\right]_{2}\left[\mathrm{Hg}_{4} \mathrm{I}_{10}\right]$ (3), entstanden durch Diffusion von methanolischen Lösungen der Metalliodide $\mathrm{HgI}_{2}$ und LiI und des Liganden (alle $\mathrm{c}=0,005 \mathrm{~mol} / \mathrm{l}$ ) ineinander. Nach einer Woche hatten sich gelbliche Kristalle gebildet, die aus Methanol umkristallsiert wurden. 


\begin{tabular}{|c|c|c|c|}
\hline & $\mathbf{1}$ & 2 & 3 \\
\hline Formel & {$\left[\left(\mathrm{CH}_{3}\right)_{4} \mathrm{~N}\right]_{2}\left[\mathrm{HgI}_{4}\right]$} & {$\left[\left(\mathrm{CH}_{3}\right)_{4} \mathrm{~N}\right]\left[\mathrm{Hg}_{2} \mathrm{I}_{5}\right]$} & {$\left[\left(\mathrm{C}_{8} \mathrm{H}_{16} \mathrm{O}_{4}\right)_{2} \mathrm{Li}\right]_{2}\left[\mathrm{Hg}_{4} \mathrm{I}_{10}\right]$} \\
\hline $\mathbf{M}_{r}$ & 856,48 & 1109,83 & 2790,14 \\
\hline Kristallsystem & orthorhombisch & orthorhombisch & monoklin \\
\hline Raumgruppe & $\begin{array}{l}P 2_{1} 2_{1} 2_{1} \text { (Nr. 19) } \\
\text { (Inversions- } \\
\text { verzwillingung) }\end{array}$ & $P 2_{1} / c 2_{1} / a 2_{1} / b($ Nr. 61$)$ & $P 2_{1} / c$ (Nr. 14) \\
\hline$a[\mathrm{pm}]$ & $968,77(3)$ & $1383,71(3)$ & $836,25(2)$ \\
\hline$b[\mathrm{pm}]$ & $1336,27(3)$ & $1455,32(1)$ & $1448,33(4)$ \\
\hline$c[\mathrm{pm}]$ & $1679,30(5)$ & $1797,69(3)$ & $2714,83(8)$ \\
\hline$\beta\left[^{\circ}\right]$ & 90 & 90 & $93,92(1)$ \\
\hline$V\left[10^{6} \mathrm{pm}^{3}\right]$ & 2174(1) & $3620(1)$ & $3280(2)$ \\
\hline$Z$ & 4 & 8 & 2 \\
\hline$D_{\text {ber }}\left[\mathrm{g} / \mathrm{cm}^{3}\right]$ & 2,62 & 4,07 & 2,83 \\
\hline Kristallgröße [mm] & $0,20 \times 0,25 \times 0,30$ & $0,35 \times 0,30 \times 0,25$ & $0,52 \times 0,25 \times 0,20$ \\
\hline $2 \theta_{\max }$ & $50^{\circ}$ & $50^{\circ}$ & $55^{\circ}$ \\
\hline Reflexzahl (unabh.) & 3807 & 3088 & 7470 \\
\hline $\begin{array}{l}\text { Lin. Absorpt.-Koeff. } \\
{\left[\mathrm{mm}^{-1}\right]}\end{array}$ & 12,8 & 25,4 & 14,1 \\
\hline Parameterzahl & 95 & 114 & 289 \\
\hline$R[I>2 \sigma(I)]$ & 0,0609 & 0,0657 & 0,0543 \\
\hline Reflexzahl $[I>2 \sigma(I)]$ & 2432 & 2123 & 3881 \\
\hline$R_{\mathrm{W}}[I>2 \sigma(I)]$ & 0,1605 & 0,1404 & 0,1096 \\
\hline $\begin{array}{l}\text { Restelektronendichte, } \\
\max . / \mathrm{min} .\left[\mathrm{e} \cdot \mathrm{pm}^{-3} \cdot 10^{-6}\right]\end{array}$ & $0,85 /-1,22$ & $2,52 /-1,58$ & $0,81 /-1,61$ \\
\hline
\end{tabular}

Tab. 1. Kristallstrukturdaten.

Tab. 3. Ausgewählte Bindungslängen (pm) und -winkel $\left(^{\circ}\right)$ in 2 .

\begin{tabular}{llll}
\hline $\mathrm{Hg}(1)-\mathrm{I}(1)$ & $309,2(2)$ & $\mathrm{Hg}(1)-\mathrm{I}(2)$ & $263,24(18)$ \\
$\mathrm{Hg}(1)-\mathrm{I}(3)$ & $263,41(18)$ & $\mathrm{Hg}(1)-\mathrm{I}(4)$ & $302,47(18)$ \\
$\mathrm{Hg}(2)-\mathrm{I}(1)^{\mathrm{ii}}$ & $274,9(2)$ & $\mathrm{Hg}(2)-\mathrm{I}(4)$ & $274,75(19)$ \\
$\mathrm{Hg}(2)-\mathrm{I}(5)$ & $272,2(2)$ & $\mathrm{Hg}(2)-\mathrm{I}(5)^{\mathrm{i}}$ & $307,7(2)$ \\
$\mathrm{I}(1)-\mathrm{Hg}(1)-\mathrm{I}(2)$ & $102,96(6)$ & $\mathrm{I}(1)-\mathrm{Hg}(1)-\mathrm{I}(3)$ & $101,30(6)$ \\
$\mathrm{I}(1)-\mathrm{Hg}(1)-\mathrm{I}(4)$ & $84,98(5)$ & $\mathrm{I}(2)-\mathrm{Hg}(1)-\mathrm{I}(3)$ & $142,38(8)$ \\
$\mathrm{I}(2)-\mathrm{Hg}(1)-\mathrm{I}(4)$ & $108,45(6)$ & $\mathrm{I}(3)-\mathrm{Hg}(1)-\mathrm{I}(4)$ & $101,91(6)$ \\
$\mathrm{I}(1)^{\mathrm{ii}}-\mathrm{Hg}(2)-\mathrm{I}(4)$ & $103,69(6)$ & $\mathrm{I}(1)^{\mathrm{ii}}-\mathrm{Hg}(2)-\mathrm{I}(5) 2$ & $103,44(7)$ \\
$\mathrm{I}(1)^{\mathrm{i}}-\mathrm{Hg}(2)-\mathrm{I}(5)^{\mathrm{i}}$ & $123,50(8)$ & $\mathrm{I}(4)-\mathrm{Hg}(2)-\mathrm{I}(5)$ & $122,18(7)$ \\
$\mathrm{I}(4)-\mathrm{Hg}(2)-\mathrm{I}(5)^{\mathrm{i}}$ & $106,97(7)$ & $\mathrm{I}(5)-\mathrm{Hg}(2)-\mathrm{I}(5)^{\mathrm{i}}$ & $93,51(6)$ \\
$\mathrm{Hg}(1)-\mathrm{I}(1)^{\mathrm{ii}}-\mathrm{Hg}(2)$ & $103,68(6)$ & $\mathrm{Hg}(1)-\mathrm{I}(4)-\mathrm{Hg}(2)$ & $90,67(6)$ \\
$\mathrm{Hg}(2)-\mathrm{I}(5)-\mathrm{Hg}(2)^{\mathrm{i}}$ & $86,49(6)$ & & \\
\hline
\end{tabular}

Tab. 4. Ausgewählte Bindungslängen (pm) und -winkel $\left(^{\circ}\right)$ in 3.

\begin{tabular}{lrll}
\hline $\mathrm{Hg}(1)-\mathrm{I}(1)$ & $306,60(10)$ & $\mathrm{Hg}(1)-\mathrm{I}(2)$ & $318,88(10)$ \\
$\mathrm{Hg}(1)-\mathrm{I}(4)$ & $262,86(11)$ & $\mathrm{Hg}(1)-\mathrm{I}(5)$ & $264,02(11)$ \\
$\mathrm{Hg}(2)-\mathrm{I}(1)$ & $276,98(11)$ & $\mathrm{Hg}(2)-\mathrm{I}(2)$ & $274,33(11)$ \\
$\mathrm{Hg}(2)-\mathrm{I}(3)$ & $269,84(10)$ & $\mathrm{Hg}(2)-\mathrm{I}(3)^{\mathrm{i}}$ & $309,88(11)$ \\
$\mathrm{I}(1)-\mathrm{Hg}(1)-\mathrm{I}(2)$ & $87,08(3)$ & $\mathrm{I}(1)-\mathrm{Hg}(1)-\mathrm{I}(4)$ & $107,93(3)$ \\
$\mathrm{I}(1)-\mathrm{Hg}(1)-\mathrm{I}(5)$ & $101,72(3)$ & $\mathrm{I}(4)-\mathrm{Hg}(1)-\mathrm{I}(5)$ & $142,08(4)$ \\
$\mathrm{I}(1)-\mathrm{Hg}(2)-\mathrm{I}(2)$ & $102,83(3)$ & $\mathrm{I}(1)-\mathrm{Hg}(2)-\mathrm{I}(3)$ & $122,33(4)$ \\
$\mathrm{I}(1)-\mathrm{Hg}(2)-\mathrm{I}(3)^{\mathrm{i}}$ & $107,77(3)$ & $\mathrm{I}(2)-\mathrm{Hg}(2)-\mathrm{I}(3)^{\mathrm{i}}$ & $92,37(3)$ \\
$\mathrm{I}(2)-\mathrm{Hg}(2)-\mathrm{I}(3)$ & $124,99(4)$ & $\mathrm{I}(3)-\mathrm{Hg}(2)-\mathrm{I}(3)^{\mathrm{i}}$ & $92,37(3)$ \\
$\mathrm{Hg}(1)-\mathrm{I}(1)-\mathrm{Hg}(2)$ & $80,47(3)$ & $\mathrm{Hg}(1)-\mathrm{I}(2)-\mathrm{Hg}(2)$ & $78,68(3)$ \\
$\mathrm{Hg}(2)-\mathrm{I}(3)-\mathrm{Hg}(2)^{\mathrm{i}}$ & $87,63(3)$ & & \\
\hline
\end{tabular}

Die Verbindungen wurden durch Röntgenstrukturanalyse charakterisiert, $\mathbf{1}$ und $\mathbf{2}$ auch durch Raman-Spektroskopie.
Die Strukturanalysen wurden unter Verwendung eines Diffraktometers Siemens SMART CCD mit Mo- $\mathrm{K}_{\alpha}$-Strahlung (Graphitmonochromator) durchgeführt. Die vorläufige Bestimmung der Gitterparameter erfolgte aus 45 „Frames“ (Schrittweite $0,3^{\circ}$ in $\omega$ ); die endgültigen Werte wurden durch Verfeinerung der Reflexe, die aus der Integration aller FrameDaten erhalten wurde, ermittelt. Die Messung erfolgte bei Raumtemperatur mit einer Schrittweite von $0,3^{\circ}$ in $\omega$, einer Zählzeit von $5 \mathrm{~s}$ pro Frame und einem Kristall-DetektorAbstand von $3 \mathrm{~cm}$ unter Verwendung des Messprogramms SMART [16]. Die Auswertung erfolgte mit dem Programm SAINT [17]. Es wurden empirische Absorptionskorrekturen (SADABS [18]) durchgeführt. Die Strukturlösung erfolgte mit direkten Methoden (SHELXS [19]), die Verfeinerung mit SHELXTL [20]. Die Positionen der Wasserstoffatome an den Liganden wurden berechnet. Abbildungen wurden mit dem Programm DIAMOND [21] erstellt. Die wichtigsten Kristallstrukturdaten sind in Tab. 1, ausgewählte Bindungslängen und -winkel in Tab. 2-4 zusammengestellt*.

\section{Ergebnisse}

Die Struktur von Bis-tetramethylammonium-tetraiodomercurat(II), $\left[\mathrm{Me}_{4} \mathrm{~N}_{2}\left[\mathrm{HgI}_{4}\right]\right.$ (1) ist in Abb. 1 dar-

\footnotetext{
*Kristallographische Daten zu den Strukturbestimmungen wurden beim Cambride Data Center unter den Hinterlegungsnummern CCDC-600913 (1), -600912 (2) und -600914 (3) hinterlegt und können kostenlos angefordert werden vom CCDC, 12 Union Road, Cambride CB2 1EZ, UK [Fax: int. Code +44(1223)3 36 033, E-mail: deposit@ccdc.cam.ac.uk].
} 


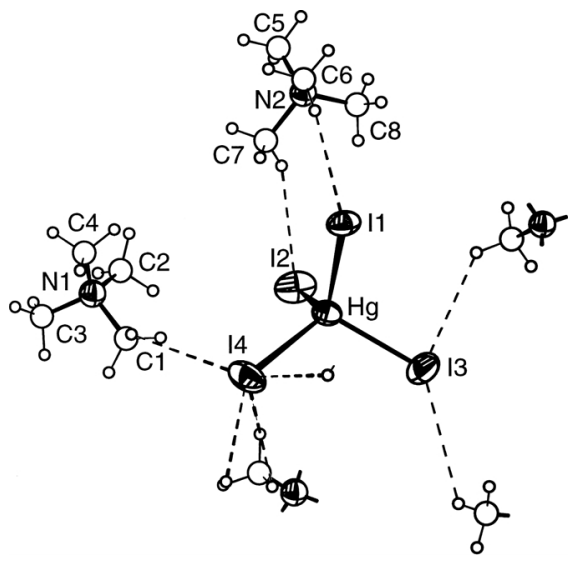

Abb. 1. Kristallstruktur von $\left[\mathrm{Me}_{4} \mathrm{~N}_{2}\left[\mathrm{HgI}_{4}\right]\right.$ (1). Wasserstoffbindungen $\mathrm{zu}$ benachbarten Tetramethylammoniumionen eingezeichnet. $\mathrm{C}, \mathrm{H}$ und $\mathrm{N}$ mit willkürlichen Radien.

gestellt. 1 kristallisiert orthorhombisch, Raumgruppe $P 2{ }_{1} 2_{1} 2_{1}$ mit 4 Formeleinheiten in der Elementarzelle. Es liegt Inversionsverzwillingung vor. Die Verbindung ist aus diskreten Tetramethylammonium- und Tetraiodomercurat-Ionen aufgebaut. Zwischen den HAtomen der Kationen und den I-Ionen der Anionen bestehen im Kristall Wasserstoffbindungen. Die kürzesten I $\cdots$ H-Abstände liegen zwischen 305 und 308 pm. Dies sind Abstände, wie sie z. B. zwischen Iodidionen und H-Atomen von OH-Gruppen und Wassermolekülen in Dicalcium-aluminium-hydroxid-iodid-Dihydrat, $\mathrm{Ca}_{2} \mathrm{Al}(\mathrm{OH})_{6} \mathrm{I} \cdot 2 \mathrm{H}_{2} \mathrm{O}$ [22] gefunden werden.

$\mathrm{Im}$ Vergleich $\mathrm{zu}$ anderen Tetraiodomercuraten ist das $\left[\mathrm{HgI}_{4}\right]^{2-}$-Ion in $\mathbf{1} \mathrm{sehr}$ regelmäßig gebaut, die Hg-I-Bindungen variieren lediglich zwischen 276,3 und 278,1 pm, ihr Mittelwert beträgt 277,3(6) pm. Dagegen liegen die Hg-I-Bindungsabstände z.B. in $\mathrm{Cs}_{2} \mathrm{HgI}_{4}$ zwischen 273 und $282 \mathrm{pm}$ [11], in $\left[\mathrm{La}_{6}(\mathrm{OH})_{8}(\mathrm{O})\left(\mathrm{H}_{2} \mathrm{O}\right)_{24}\right]\left[\mathrm{HgI}_{4}\right]$ zwischen 273 und 289 pm [8]. Die Bindungswinkel I-Hg-I in 1 zeigen relativ geringe Abweichungen vom idealen Tetraederwinkel, während sie in der Lanthanverbindung zwischen 103 und $120^{\circ}$ liegen. Die leichte Verzerrung des Tetraeders in $\mathbf{1}$ ist wahrscheinlich durch Packungseffekte und die relativ geringen Wechselwirkungen mit den Kationen bedingt. Die [ $\left.\mathrm{Me}_{4} \mathrm{~N}\right]-$ Gruppen weisen eine geringe Fehlordnung auf, was sich in hohen Auslenkungsparametern äußert. Sie wurden daher nur isotrop und mit Constraints für die N-C-Bindungslängen verfeinert.

Tetramethylammonium-pentaiododimercurat(II), $\left[\mathrm{Me}_{4} \mathrm{~N}\right]\left[\mathrm{Hg}_{2} \mathrm{I}_{5}\right]$ (2), kristallisiert orthorhombisch,

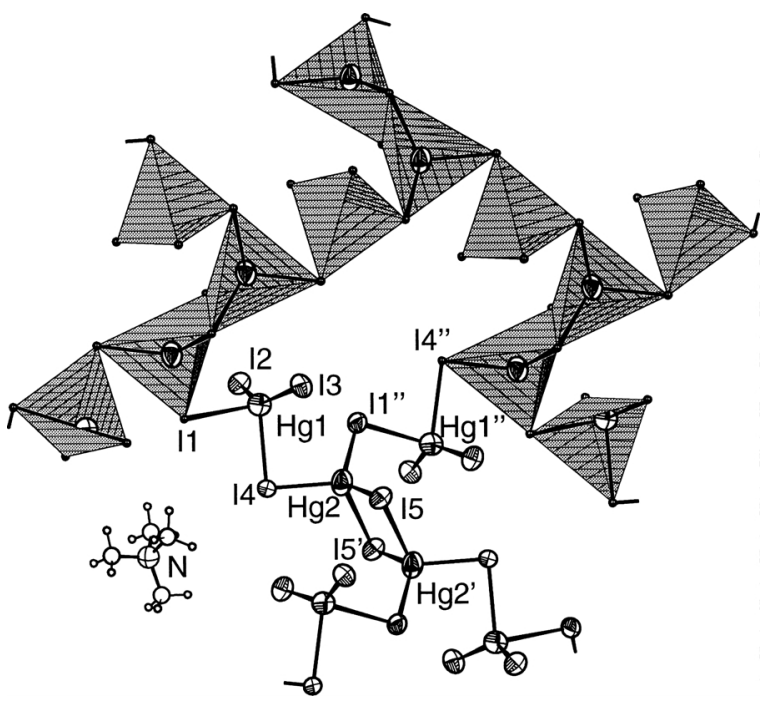

Abb. 2. Ausschnitt aus der Schichtstruktur von $\left[\mathrm{Me}_{4} \mathrm{~N}\right]-$ $\left[\mathrm{Hg}_{2} \mathrm{I}_{5}\right]$ (2). Um die Verknüpfung zu verdeutlichen, sind einige $\left[\mathrm{HgI}_{4}\right]$-Einheiten als Polyeder eingezeichnet. Symmetriecode: $\mathrm{i}=2-x, 1-y,-z ; \mathrm{ii}=0,5+x, 1,5-y, z$.

Raumgruppe Pcab (Nichtstandardaufstellung von $\mathrm{Pbca}$, Nr. 61), mit 8 Formeleinheiten in der Elementarzelle. Die Verbindung ist im Kristall aus $\left[\mathrm{Me}_{4} \mathrm{~N}\right]^{+}$-Kationen und polymeren $\left[\mathrm{Hg}_{2} \mathrm{I}_{5}\right]_{\mathrm{n}}{ }^{\mathrm{n}+}$ Anionen aufgebaut (s. Abb. 2). Die Anionen bilden Schichten, die parallel zur $a b$-Ebene im Kristall liegen. In diesen Schichten sind jeweils zwei $\left[\mathrm{HgI}_{4}\right]$-Tetraeder über eine gemeinsame Kante $\mathrm{zu}$ $\left[\mathrm{Hg}_{2} \mathrm{I}_{6}\right]$-Einheiten verknüpft; diese Einheiten werden mit $\left[\mathrm{HgI}_{4}\right]$-Tetraedern über gemeinsame Ecken verbrückt, wobei die Verknüpfung an allen freien Ecken der $\left[\mathrm{Hg}_{2} \mathrm{I}_{6}\right]$-Einheiten erfolgt. Die $\left[\mathrm{Hg}_{2} \mathrm{I}_{6}\right]$-Spezies sind alternierend senkrecht zueinander angeordnet. Es entstehen so ringförmige Elemente mit zehn $\mathrm{Hg}$ Atomen, wobei jede zweite $\left[\mathrm{Hg}_{2} \mathrm{I}_{6}\right]$-Einheit mit einem Hg-Atom diesem Ring, mit dem zweiten Hg-Atom einem benachbarten, anellierten Ring angehört. In der Abb. 2 ist ein Ausschnitt aus der Schicht mit einem Ring und verbrückenden $\left[\mathrm{HgI}_{4}\right]$-Tetraedern dargestellt. Das $\left[\mathrm{Hg}_{2} \mathrm{I}_{5}\right]$-Anion in (2) unterscheidet sich damit grundlegend von dem von uns an anderer Stelle [8] beschriebenen kettenförmigen $\left[\mathrm{Hg}_{2} \mathrm{I}_{5}\right]$-Anion in [K(benzo-15-krone-5) $\left.{ }_{2}\right]\left[\mathrm{Hg}_{2} \mathrm{I}_{5}\right]$, bei dem die $\left[\mathrm{Hg}_{2} \mathrm{I}_{6}\right]$ Einheiten nicht über $\left[\mathrm{HgI}_{4}\right]$-Tetraeder verknüpft sind, sondern die Hg-Atome benachbarter Einheiten jeweils über gemeinsame $\mu_{2}$-verbrückende Iodidionen verbunden sind. Die $\mathrm{Hg}$-I-Bindungsabstände in $\mathbf{2}$ variieren stark. Am kürzesten sind mit $c a .275$ pm 
die terminalen $\mathrm{Hg}(1)-\mathrm{I}-\mathrm{Bindungen}$ der verbrückenden

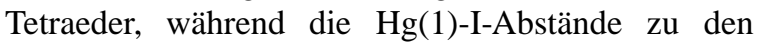
brückenbildenden I-Atomen mit 302 und 309 pm signifikant länger sind, so dass die Tetraeder erheblich verzerrt werden. Diese Verzerrung zeigt sich noch stärker bei den Bindungswinkeln. Der Winkel zu den terminalen Iodidionen, $\mathrm{I}(2)-\mathrm{Hg}(1)-\mathrm{I}(3)$, ist mit $142^{\circ}$ stark aufgeweitet, während der Winkel $\mathrm{I}(1)-\mathrm{Hg}(1)-\mathrm{I}(4)$ mit den verbrückenden Iodidionen mit $85^{\circ}$ entsprechend verkleinert ist. Die $\left[\mathrm{Hg}_{2} \mathrm{I}_{6}\right]-$ Einheiten weisen zwar ein Symmetriezentrum auf, die Hg-I-Bindungen zwischen den beiden symmetrieäquivalenten $\mathrm{Hg}$-Atomen $\mathrm{Hg}(2)$ und $\mathrm{Hg}(2)^{\mathrm{i}}$ sind jedoch sehr unsymmetrisch: so ist die Bindung $\mathrm{Hg}(2)-\mathrm{I}(5) \mathrm{mit}$ 272 pm recht kurz, $\mathrm{Hg}(2)-\mathrm{I}(5)^{\mathrm{i}}$ dagegen mit 308 pm sehr viel länger. Die ,quasiterminalen“ Bindungen zwischen $\operatorname{Hg}(2)$ und $\mathrm{I}(1)^{\mathrm{ii}}$ bzw. I(4) sind in etwa gleich lang und liegen mit 275 pm im Bereich der kürzeren Bindungslängen. Im kettenförmigen Anion in $\left.[\mathrm{K} \text { (benzo-15-krone-5) })_{2}\right]\left[\mathrm{Hg}_{2} \mathrm{I}_{5}\right]$, in dem ebenfalls zentrosymmetrische $\left[\mathrm{Hg}_{2} \mathrm{I}_{6}\right]$-Einheiten vorliegen, sind diese jedoch sehr viel symmetrischer, die beiden Hg-I-Abstände liegen bei 288 pm und unterscheiden sich nur um 0,5 pm.

Die $\left[\mathrm{Me}_{4} \mathrm{~N}\right]-$ Gruppen liegen in den durch die Ringe resultierenden Hohlräumen. Die Gruppen sind ebenfalls geringfügig fehlgeordnet. Stärkere Wechselwirkungen zwischen den $\mathrm{H}$ - und den I-Atomen bestehen nicht, der kürzeste I $\cdots \mathrm{H}$-Abstand beträgt $c a .323 \mathrm{pm}$.

Bis[di(12-krone-4)lithium]decaiodotetramercurat(II) [Li(12-krone-4) $\left.{ }_{2}\right]_{2}\left[\mathrm{Hg}_{4} \mathrm{I}_{10}\right](3)$, kristallisiert monoklin in der zentrosymmetrischen Raumgruppe $P 2_{1} / c$ (Nr. 14), mit 2 Formeleinheiten in der Elementarzelle. Im Kristall liegen gestreckte $\left[\mathrm{Hg}_{4} \mathrm{I}_{10}\right]^{2-}$-Anionen und durch je zwei Kronenethermoleküle komple-

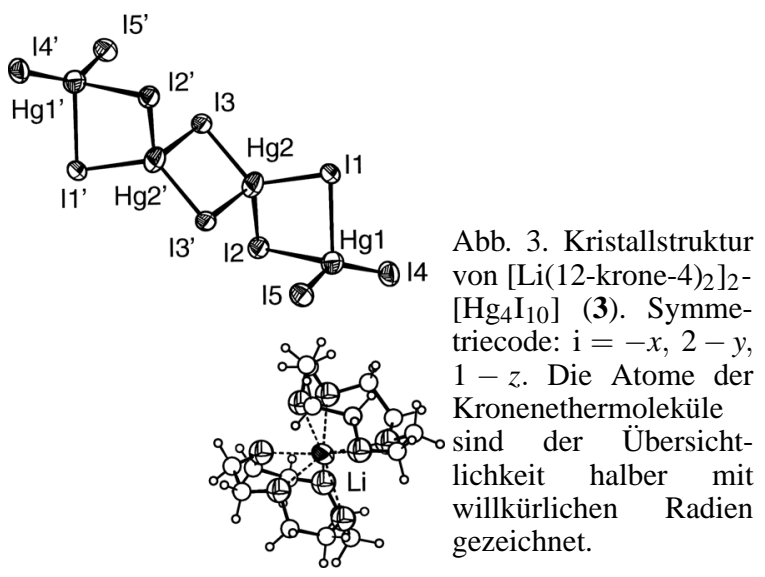

xierte $\mathrm{Li}^{+}$-Kationen vor (s. Abb. 3). Das Anion besitzt ein Symmetriezentrum, es ist aus vier kantenverknüpften $\left[\mathrm{HgI}_{4}\right]$-Tetraedern aufgebaut. Die terminalen $\mathrm{Hg}$-I-Bindungen sind etwa gleich lang, mit 263 pm sehr kurz und auch deutlich kürzer als die $\mathrm{Hg}$-I-Abstände im $\left[\mathrm{HgI}_{4}\right]$-Ion selbst (s. Verb. 1), die beiden Brückenbindungen im Tetraeder um $\mathrm{Hg}(1)$ sind dagegen mit 307 und 319 pm stark aufgeweitet. Die ,inneren“ Tetraeder sind stark verzerrt, die Bindungen sind unsymmetrisch: der Abstand von $\mathrm{Hg}(2) \mathrm{zu} \mathrm{I}(3)$ beträgt $270 \mathrm{pm}$, zum symmetrieäquivalenten $\mathrm{I}(3)^{\mathrm{i}} 310 \mathrm{pm}$. In dem von Nockemann et al. [7] beschriebenen Bis(tetrabutylammonium)decaiodotetramercurat(II) ist das $\left[\mathrm{Hg}_{4} \mathrm{I}_{10}\right]^{2-}$-Ion analog aufgebaut, es ist ebenfalls gestreckt, weist aber kein Symmetriezentrum auf. Die Abfolge kurzer und langer Bindungsabstände ist ähnlich, nur variieren die $\mathrm{Hg}$-I-Abstände noch stärker als die in $\mathbf{3}$, ihr Bereich beträgt 261 bis $331 \mathrm{pm}$.

\section{Diskussion}

Abgesehen vom Triiodomercurat(II)-Ion, in dem die Quecksilberionen eine trigonal-bipyramidale Koordination aufweisen, sind die Quecksilberatome in den meisten Iodomercuraten tetraedrisch koordiniert. In Tab. 5 sind die bisher bekannten „Klassen“ von Iodomercuraten zusammengestellt. In Anlehnung an die Bezeichnungen in der Silicatchemie haben wir sie in Neso(Insel)-, Soro(Gruppen)-, Ino(Ketten)- und Phyllo(Schicht)-Mercurate eingeteilt, obwohl die Verhältnisse bei Silicat-Anionen etwas anders liegen: zwar sind auch hier die Baugruppen Tetraeder, jedoch können diese im Gegensatz zu den Mercuraten nicht kantenverknüpft sein, sondern sind stets eckenverknüpft. Bei den Mercuraten kommen beide Verknüpfungsarten vor. Reine Kantenverknüpfung führt zu Ionen der $\mathrm{Zu}$ sammensetzung $\left[\mathrm{Hg}_{\mathrm{n}} \mathrm{I}_{2 \mathrm{n}+2}\right]^{2-}$, die bis $\mathrm{n}=4$ dargestellt wurden. Bei $\left[\mathrm{Hg}_{3} \mathrm{I}_{8}\right]^{2-}$ sind zwei Isomere mit verschiedenen Verknüpfungen bekannt. Ausgehend von der Einheit $\left[\mathrm{Hg}_{2} \mathrm{I}_{6}\right]^{2-}$ aus zwei kantenverknüpften Tetraedern werden durch direkte Eckenverknüpfung derartiger Einheiten polymere, kettenförmige $\left[\mathrm{Hg}_{2} \mathrm{I}_{5}\right]^{-}$Ionen gebildet, durch Eckenverknüpfung über weitere $\left[\mathrm{HgI}_{4}\right]$-Tetraeder $\left[\mathrm{Hg}_{2} \mathrm{I}_{5}\right]^{-}$-Ionen mit Schichtstruktur. Tekto(Gerüst)-Mercurate existieren nicht, rotes $\mathrm{HgI}_{2}$ besteht jedoch aus einem Netzwerk eckenverknüpfter Tetraeder [24]. In Mercuraten wie $\mathrm{Cu}_{2} \mathrm{HgI}_{4}$ liegt ebenfalls ein Netzwerk aus eckenverknüpften Tetra- 
Tab. 5. Zusammenstellung der bisher bekannten Klassen von Iodomercurat(II)-Ionen.

\begin{tabular}{|c|c|c|c|c|}
\hline Klasse & $\begin{array}{l}\text { Formale } \\
\text { Zusammen- } \\
\text { setzung }\end{array}$ & Aufbau & Beispiele & Ref. \\
\hline Nesomercurate & {$\left[\mathrm{HgI}_{4}\right]^{2-}$} & $\begin{array}{l}\text { diskrete tetraedrische } \\
{\left[\mathrm{HgI}_{4}\right]^{2-} \text {-Ionen }}\end{array}$ & $\begin{array}{l}{\left[\mathrm{Me}_{3} \mathrm{~S}_{2}\left[\mathrm{HgI}_{4}\right]\right.} \\
\mathrm{Cs}_{2}\left[\mathrm{HgI}_{4}\right] \\
{\left[\mathrm{Me}_{4} \mathrm{~N}_{2}\left[\mathrm{HgI}_{4}\right]\right.}\end{array}$ & $\begin{array}{l}{[23]} \\
{[11]} \\
\text { diese Arbeit }\end{array}$ \\
\hline \multirow[t]{3}{*}{ Soromercurate } & {$\left[\mathrm{Hg}_{2} \mathrm{I}_{6}\right]^{2-}$} & 2 kantenverknüpfte $\left[\mathrm{HgI}_{4}\right]$-Tetraeder & $\begin{array}{l}{[\mathrm{Cs}(15-\mathrm{krone}-5)]_{2-}\left[\mathrm{Hg}_{2} \mathrm{I}_{6}\right]} \\
{\left[\mathrm{HgI}(\text { diaza-15-krone-5) }]\left[\mathrm{Hg}_{2} \mathrm{I}_{6}\right]\right.}\end{array}$ & $\begin{array}{l}{[6]} \\
{[14]}\end{array}$ \\
\hline & {$\left[\mathrm{Hg}_{3} \mathrm{I}_{8}\right]^{2-}$} & $\begin{array}{l}3 \text { kantenverknüpfte }\left[\mathrm{HgL}_{4}\right] \text {-Tetraeder: cis-verküpft oder } \\
\text { trans-verknüpft }\end{array}$ & $\begin{array}{l}{[\mathrm{Hg}(\mathrm{kryptand} 222)]-\left[\mathrm{Hg}_{3} \mathrm{I}_{8}\right]} \\
{\left[\mathrm{Li}(12-\mathrm{krone}-4)_{2}\right]_{2-}\left[\mathrm{Hg}_{3} \mathrm{I}_{8}\right]}\end{array}$ & $\begin{array}{l}{[1]} \\
{[8]}\end{array}$ \\
\hline & {$\left[\mathrm{Hg}_{4} \mathrm{I}_{10}\right]^{2-}$} & 4 kantenverknüpfte $\left[\mathrm{HgL}_{4}\right]$-Tetraeder & $\begin{array}{l}{\left[\left(t-\mathrm{bu}_{4} \mathrm{~N}\right)_{2}\right]\left[\mathrm{Hg}_{4} \mathrm{I}_{10}\right]} \\
{\left[\mathrm{Li}(12-\mathrm{krone}-4)_{2}\right]_{2}\left[\mathrm{Hg}_{4} \mathrm{I}_{10}\right]}\end{array}$ & $\begin{array}{l}{[7]} \\
\text { diese Arbeit }\end{array}$ \\
\hline \multirow[t]{2}{*}{ Inomercurate } & {$\left[\mathrm{HgI}_{3}\right]^{-}$} & $\begin{array}{l}\text { über Hg-I-Wechselwirkungen zu Ketten verknüpft, } \mathrm{Hg} \\
\text { trigonal-bipyramidal koordiniert }\end{array}$ & {$\left[\mathrm{Me}_{3} \mathrm{~S}\right]\left[\mathrm{HgI}_{3}\right]$} & [9] \\
\hline & {$\left[\mathrm{Hg}_{2} \mathrm{I}_{5}\right]^{2-}$} & $\begin{array}{l}{\left[\mathrm{Hg}_{2} \mathrm{I}_{6}\right] \text {-Einheiten aus kantenverknüpften }\left[\mathrm{HgL}_{4}\right] \text {-Tetra- }} \\
\text { edern trans-ständig zu Ketten verknüpft }\end{array}$ & $\operatorname{Di}\left[(\right.$ benzo-15-krone-5) $\mathrm{K}]\left[\mathrm{Hg}_{2} \mathrm{I}_{5}\right]$ & [8] \\
\hline Phyllomercurate & {$\left[\mathrm{Hg}_{3} \mathrm{I}_{8}\right]^{2-}$} & Schichten aus eckenverknüpften $\left[\mathrm{HgL}_{4}\right]$-Tetraedern & $\mathrm{Cs}_{2}\left[\mathrm{Hg}_{3} \mathrm{I}_{8}\right] \cdot \mathrm{H}_{2} \mathrm{O}$ & [11] \\
\hline & {$\left[\mathrm{Hg}_{2} \mathrm{I}_{5}\right]^{2-}$} & $\begin{array}{l}{\left[\mathrm{Hg}_{2} \mathrm{I}_{6}\right] \text {-Einheiten aus kantenverknüpften }\left[\mathrm{HgL}_{4}\right]-} \\
\text { Tetraedern an jeder Ecke über }\left[\mathrm{HgI}_{4}\right] \text {-Tetraeder zu } \\
\text { Schichten verknüpft }\end{array}$ & {$\left[\mathrm{Me}_{4} \mathrm{~N}\right]\left[\mathrm{Hg}_{2} \mathrm{I}_{5}\right]$} & diese Arbeit \\
\hline
\end{tabular}

edern vor, jedoch wechseln sich $\left[\mathrm{HgI}_{4}\right]$ - und $\left[\mathrm{CuI}_{4}\right]-$ Tetraeder ab [25]. Nicht berücksichtigt sind in der $\mathrm{Zu}-$ sammenstellung Mercurat-Iodide wie das von Gerken und Pakhomov beschriebene $\mathrm{K}_{2}\left[\mathrm{HgI}_{4}\right] \cdot 3 \mathrm{H}_{2} \mathrm{O}$ [26], das $\left[\mathrm{HgI}_{3}\right]$-Ionen enthält, die durch $\mathrm{I}^{-}$-Ionen verbrückt sind, sowie $\left[\mathrm{Et}_{4} \mathrm{~N}\right]_{2}\left[\mathrm{Hg}_{3} \mathrm{I}_{8}\right]$, von dem wir eine Rönt- genstrukturanalyse durchführten, die zeigte, dass hier $\left[\mathrm{Hg}_{2} \mathrm{I}_{6}\right]$-Ionen und $\mathrm{HgI}_{2}$-Einheiten vorliegen, die über Hg... I-Wechselwirkungen verknüpft sind [27]; wegen mangelhafter Kristallqualität ist die Analyse allerdings relativ ungenau, so dass wir bisher von einer Veröffentlichung absehen.
[1] J. Pickardt, B. Kühn, J. Chem. Soc., Chem. Commun. 451 (1995).

[2] L. G. Sillén, Acta Chim. Scand. 3, 539 (1949).

[3] M. Sandström, G. Johansson, Acta Chim. Scand. A31, 132 (1977).

[4] G. B. Deacon, B. O. West, Austral. J. Chem. 16, 579 (1963).

[5] M. A. Hoaper, D. W. James, Austral. J. Chem. 24, 1331 (1971).

[6] S. Wiese, Dissertation, Technische Universität Berlin (2003).

[7] P. Nockemann, G. Meyer, Acta Crystallogr. E59, m236 (2003).

[8] J. Pickardt, S. Wiese, L. v. Chrzanowski, M. Borowski, Z. Anorg. Allg. Chem. 626, 2096 (2000).

[9] R. H. Fenn, Acta Crystallogr. 20, 20 (1966).

[10] L. Nyqvist, G. Johansson, Acta Chim. Scand. 25, 1615 (1971).

[11] R. Sjövall, C. Svensson, Acta Crystallogr. C44, 207 (1988).

[12] J. Pickardt, P. Wischlinsky, Z. Anorg. Allg. Chem. 622, 1125 (1996).
[13] U. Müller, Anorganische Strukturchemie, S. 135, Teubner-Verlag, Stuttgart (1991).

[14] J. Pickardt, S. Wiese, Z. Naturforsch. 52b, 847 (1997).

[15] A. F. Wells, Structural Inorganic Chemistry, Clarendon, Oxford 1161 (1984).

[16] SMART Software Reference Manual, Version 4.0, Siemens Analytical X-Ray Instruments, Madison (WI) (1996).

[17] SAINT Software Reference Manual, Version 4.0, Siemens Analytical X-Ray Instruments, Madison (WI) (1994; 1996).

[18] G. M. Sheldrick, SADABS, Empirical Absorption Correction Program, Göttingen (1996).

[19] G. M. Sheldrick, SHELXS-97, Program for Crystal Structure Solution, Göttingen (1997).

[20] G. M. Sheldrick, SHELXTL Reference Manual, Version 5.1, Bruker AXS, Madison (WI) (1994; 1997).

[21] G. Bergerhoff, K. Brandenburg, M. Berndt, DIAMOND, Visuelles Informationssystem für Kristallstrukturen, Bonn (1996).

[22] J.-P. Rapin, A. Walcariuss, G. Levevre, M. François, Acta Crystallogr. C55, 1957 (1999). 
[23] R. H. Fenn, Acta Crystallogr. 20, 24 (1966).

[24] G. A. Jeffrey, M. Vlasse, Inorg. Chem. 6, 396 (1967).

[25] H. Hahn, G. Frank, W. Klingler, Z. Anorg. Allg. Chem. 279, 271 (1955)
[26] V. A. Gerken, V. I. Pakhomov, Zh. Strukt. Khimii 10, 753 (1969).

[27] J. Pickardt, unveröffentlicht. 